\title{
Dynamics Evolution Processes of Ecological Building Materials Industry
}

\author{
X. X. XIAO, W. LONG \\ School of economic Wuhan University of Technology Wuhan, P.R.China
}

\begin{abstract}
In this article, we discussed the dynamic development path of ecological building materials industry, suitable measures for keepings steady path, built integrated model using a patches prototype structure as part of it, optimal solution of model will be discussed and with real data obtained from statistical review and numerical simulations which show how they affect the models. Our purpose is figure out reasonable dynamic development direction for the ecological building materials industry to implementation in prospective construction.
\end{abstract}

KEYWORD: ecological building materials industry; improved model; dynamics equilibrium; evolution processes

\section{GENERAL INSTRUCTIONS}

Construction industry is one of highest resource demands, with the continuous depletion of fossil fuel reserves, the world has shifted its focus towards future resources acquisition. In this context, Ecological Building Materials has recently emerged as a significant candidate in the area sustainable materials industry. A vast amount of literature is devoted to integrated analysis on (Freeman) the basis of the relevant organization ecology, put forward the complete organization ecology concept and the research framework, set up the mathematical model of individual development, founding rate and mortality rate shows that the fluctuation of population, for the two influence factors, do not measure them directly, but be assumed to the population number-density function within the current organization. (Hnanna and Carorl, 1992) (Baunl\&Sinhg, 1994) points out that the current focus on the ecological industry organization evolution of hierarchy nature, including within the organization, the research on volution of organization at all levels. (Tilman, 1973) studied the habitat destruction under unidirectional (strong species competition and weak species) $\mathrm{n}$ - collection of population competition mechanism. (Xu cai lin, 2010) as studied competing mechanism of twopopulation system. (Zi zhen li, 2012) analyzed the two heterogeneous population fled to coexist under the patch habitat models and related issues of competition and coexistence mechanism and cooperative coevolution.
Research on The sustainable industrial growth path of the article is not much in the field of industry evolution mainly concentrated in the industrial technology progress to improve the source efficiency. we discussed the dynamic development path of ecological building materials industry, and gives the suitable measures for keepings steady path, by built integrated model using a patches prototype structure as part of them, optimal solution of model will be discussed and with real data obtained from statistical review and numerical simulations which show how they affect the models. Our purpose is figure out reasonable dynamic development direction for the ecological building materials industry to implementation in prospective construction.

\section{INTEGRATED MODELS DESCRIPTION}

A variety of complexity industry evolution can be describe the process of economic and biological dynamic progress, which exchange resource, energy and information between the external environment and industry system that affect proceeding of the evolution.

In two species industry system, the evolution tendency of the industries population is either a population trend to extinction the other trend to boom period in the progress, or two industries population to achieve some kind of coexistence.

We propose building materials industry systems including two species, ecological building materials 
industry population and general building materials industry population. Ecological building materials is emerging industry population, which from obtain legal status to high-level degree of organization is detailed and complicated process [2], thus draw into the patches model to integrated models, which including patches dynamic progress and populations who rely on patches dynamic, so it is a nonlinear dynamic model.

\subsection{The patches Model}

A basic model was originally derived to constraints and elements of patches. A later modification at continuous time models associated with two limiting elements access and patches [1] as follows:

$$
\begin{aligned}
& \frac{d R_{j}}{d t}=a_{j}\left(s_{j}-R_{j}\right)-\sum_{j=1}^{n}\left[N_{i} f\left(R_{i}\right) / Y_{i j}\right] \\
& \frac{d N_{i}}{d t}=N_{i}\left[\min _{1 \leq j \leq m}\left(f\left(R_{i}\right)-m_{i}\right)\right]
\end{aligned}
$$

\subsection{An improved model}

Overseeing the basic patches model characteristics such as patches resource heterogeneity on spatial or temporal scales and acquisition capacity of species, those elements not be involved in models, which can lead to serious misrepresentation of the Pathdependent and optimal controls. Admitting that the construction industry is assumed a single-patch for building materials industry system, but the system is not consumed resources and energy of construction industry. Distinct from the theory of the patches, considerations the patch potentially influences are necessary to model; hence an improved model is defined as follows:

$$
\begin{aligned}
& \frac{d B}{d t}=\sum_{i=1}^{n} a_{i} B_{i}\left(1-B_{i}\right)-\sum_{i=1}^{n} d_{i} B_{i} \\
& \frac{d N_{i}}{d t}=N_{i}\left(a_{i} b_{i}(B)-\sigma_{i j} N_{i}-d_{i}\right)
\end{aligned}
$$

Where B refers to the density of building industry resources and $B_{i}$ is the proportion of patches occupied by species $i . N_{i}$ denote the population density of building materials industry, $a_{i}$ is represented the diffusion rate of building material industry, and $b_{i}(B)$ denote ability to capture industry resources construction of the building materials industry $i$, Species-specific dispersal rates and mortality rates are represented by $\sigma_{i j}$ and $d_{i}$, respectively.

\subsection{The steady state analysis}

There is only a single population system is easy to reach the stable state building materials industry, and always maintain a stable state. But consider two building materials industry in the equilibrium under the environment of population in the construction industry, the model containing the unknown function. We assume that the building materials industry system made up of two populations that ecological building materials industry and general building materials industry that constitute an organic synthesis in building materials industry system, with construction industry as its single patch. For the improved model, denote the growth of the construction industry function and we make the assumptions by: Function $\mathrm{f}$ (B) and $\mathrm{B}$ (B) is continuously differentiable, if $K>0 \quad B>0, \quad f(B)>0$, And when $\mathrm{b}(\mathrm{B})>0 \mathrm{f}(\mathrm{B})>0, \mathrm{~B} \in[0, \mathrm{~K})$; when $\mathrm{f}(\mathrm{B})<0$, $\mathrm{B}>\mathrm{K}$, therefore through graphic simulation in a certain period of time of the three links command. Reference model, we put the space is decomposed into three dimensions: population density of the ecological building materials industry, population density of general building materials industry and response ability for patches resources. The simulations with more neighborhood are run 15 time steps for each of $25 \times 25$ combing parameters in a two-dimensional space of $50 \times 50$ patches. We run these simulations 20 times to maintain the reality and accuracy of the frequencies (Figure1).

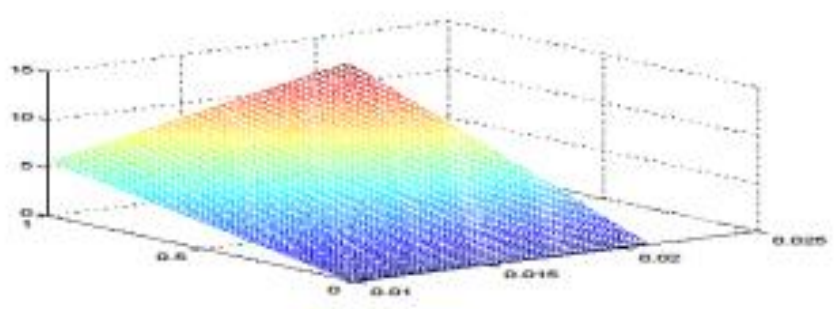

Figure1: three-dimensional of steady state

By model analysis and graphical display of the mechanism of population in the two industries, when time to change, two industry population change trend of resource patches reactions is similar, in the previous, two industries populations to patches resources growth response can be approximate. Gradually, the reaction of the ecological building materials industry becomes significantly sensitivity and the population size presents widening [4]. That also be explained from the mechanism, two of the population dynamic depends on the resources patches, increase the patches size or growth rate of resources, population of two industry can coexist in the early days, when resources growth is endless, single patch idealized infinite resource growth rate can create an unlimited number of competitive 
industrial population of long-term coexistence environment. On the contrary, in the time span long coexistence industry populations, like the building materials industry systems, the patch resources at a certain growth rate who becomes significantly sensitivity reaction ability of patches resources group, will survival be a long time.

\section{PROCESSES OF THE ECOLOGICAL BUILDING MATERIALS INDUSTRY}

\subsection{Differences of ecological building materials industry evolution}

In the model, continuous adjustments acquisition capacity of patch resources effort drive ecological building materials industry ratios toward an optimum that is assumed to be set by genetic constraints and changes only with algometry. Resource acquisition effort is constrained by the requirement of patch resources.

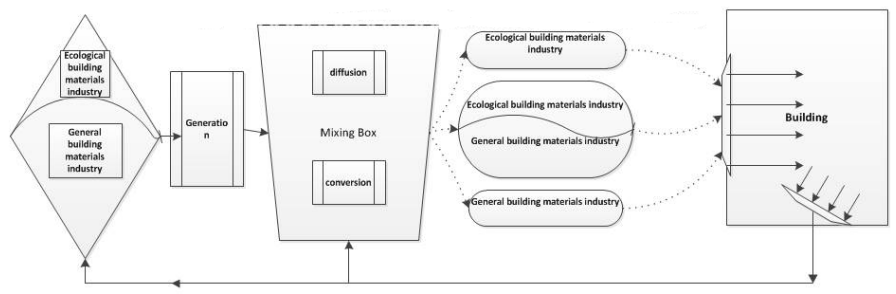

Figure 2 Dynamic processes of ecological building materials industry

Thus, the key feature of model are that populations acclimates to changes in the availability of patch resources, by continuously adjusting effort expended toward their acquisition (Figure2) assumed strongly correlated with growth of the patch .If prospective potential patches of resources have been successfully developed further, ecological building materials industry population was paying its core advantage on innovation diffusion to exploit prospective potential patches of building industry resources, especially for the key position in the industry enterprises. Therefore rapid growth of population density changes ecological building materials species genetic constraints by found new spatial diffusion, kinds of new enterprise to join the population, also transferred from the general population. Instead, the core resources of original market are fully developed with mature populations of general materials tend to be saturated, population size no longer increases even start to shrink.

\subsection{Equations Ability to exploit construction industry resource}

Analysis the related parameters are crucial because of undefined function and tougher parameter that make the process mutability in the model. According to the number of ecological building materials enterprises to estimate part of the industry population parameters in the model, definition of ecological building materials enterprise standard has not been established, the data processing is used from the environmental protection department of the People's Republic of China and the bureau of statistics of China and China industrial economic statistical yearbook data arrangement, building materials enterprise gaining environmental protection labelling certification should be supposed to ecological building materials population:

Table 1: The population quantity

\begin{tabular}{|c|c|c|c|c|c|}
\hline time quantity & 2009 & 2010 & 2011 & 2012 & 2013 \\
\hline $\begin{array}{c}\text { Ecological } \\
\text { building materials } \\
\text { industry }\end{array}$ & 507 & 864 & 1243 & 1860 & 3178 \\
\hline $\begin{array}{c}\text { Building } \\
\text { materials industry }\end{array}$ & 8982 & 10556 & 6790 & 7472 & 8372 \\
\hline
\end{tabular}

According to the Logistic evolution model parameters set parameters $\mathrm{k}$ and $\mathrm{r}$ for fixed values: 2009 as $\mathrm{t}=1$ time. Part of the model parameters were sited, Adjustments the original value of $\mathrm{Ni}$ $(i=1,2,3,4)$ and function, we have got Four species common evolution development initial period (Figure3).

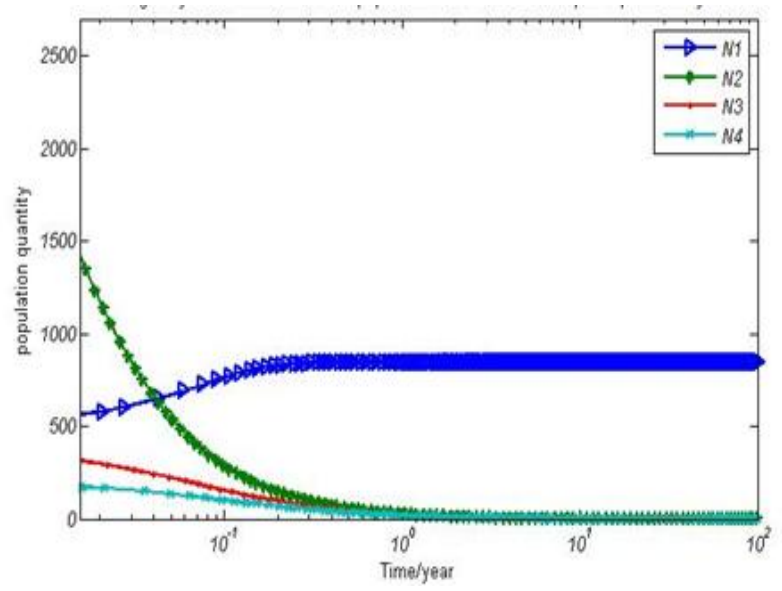

Figure 3 Dynamic evolution of four population based on different preemption ability

But at mid-late evolution process, $\mathrm{N} 1$ population density increased gradually to a steady state, evolution of the other three groups are gradually become extinct, simultaneously, the population density decrease with the add value of the ability to exploit construction industry resources showing a positive correlation, For example the extinction time of the weakest N4 is 10 year, the steady state density of population N1 is 9 year (Fig1). The numerical simulation process can be seen that in the case of other parameters are the same, different exploit industry resources function value of ability bring 
different dynamic [5]. N2 have the largest initial density of populations but been assumed the ability to exploit construction resource is less than the N1, after a period of time reducing the degree of original population advantage, finally tend to be extinct with $\mathrm{N} 3$ and N4. The simulation illustrates the role of ability to exploit construction resource under the industry evolution, so ecological building materials industry population need strong preemption ability to compensate for weaker initial population density to steadily evolved in patches environment.

\section{CONCLUSIONS}

The same conclusion is obtained by repeated longer simulation, So the preemption ability of industry population play a key role under patch resources [6], the influence of preemptiion and diffusion ability is different between difference population. Although the larger initial density of populations the stronger diffusion ability has, In the process of evolution strong population density increasing, while density of smaller populations has Large range of fluctuations, but by increasing preemptiion ability to the growing construction resources patches, its density can also stability along the growth path at one moment two industry population been balanced, so two kinds of population in the same industry system stable coexistence is achievable.

Just in the midst of the early industrial development of ecological building materials industry under the condition of smaller population density can be long-term stability along the growth path of evolution, the point is preemptive ability to the increasing construction resources patches.

\section{REFERENCES}

[1] O.Flaaten, Bioeconomics of sustainable harvest of competing species, Journal of Environmental Economics and Management pp.163-180, 1991.

[2] E References.H. Bulte a,G.C. van Kooten, Metapopulation dynamics and stochastic bioeconomic modelling, Ecological Economics pp.293-299, 1999.

[3] C.V. Pezzey, C.M. Roberts, B.T. Urdal, A simple bioeconomic model of a marine reserve, Ecological Economics, pp. 77-91, 2000.

[4] Kallis G.When is it coevolution. Ecological Economics, pp.1-6, 2007.

[5] SuhomlinovaO. Toward a model of organizational evolution in transition economies. Joumal of Management Studies. 2006, pp.1537-1558.

[6] GaravagliaC., Breschi S. The Co-evolution of entrepreneurships and Clusters, Journal, (Issue): pp.95-116, 2009. 